\title{
Decreased pancreatic acinar cell number in type 1 diabetes
}

\section{Jordan J. Wright ${ }^{1}$ (D) - Diane C. Saunders ${ }^{1}$ (D) $\cdot$ Chunhua Dai ${ }^{1} \cdot$ Greg Poffenberger ${ }^{1} \cdot$ Brynn Cairns $^{2} \cdot$ David V. Serreze $^{2}$. David M. Harlan ${ }^{3}$ (D) $\cdot$ Rita Bottino ${ }^{4}$ (D) $\cdot$ Marcela Brissova ${ }^{1}$ (D) $\cdot$ Alvin C. Powers ${ }^{1,5,6}$ (I)}

Received: 13 November 2019 / Accepted: 20 March 2020 / Published online: 9 May 2020

(C) Springer-Verlag GmbH Germany, part of Springer Nature 2020

\begin{abstract}
Aims/hypothesis Individuals with longstanding and recent-onset type 1 diabetes have a smaller pancreas. Since beta cells represent a very small portion of the pancreas, the loss of pancreas volume in diabetes is primarily due to the loss of pancreatic exocrine mass. However, the structural changes in the exocrine pancreas in diabetes are not well understood.

Methods To characterise the pancreatic endocrine and exocrine compartments in diabetes, we studied pancreases from adult donors with type 1 diabetes compared with similarly aged donors without diabetes. Islet cell mass, islet morphometry, exocrine mass, acinar cell size and number and pancreas fibrosis were assessed by immunohistochemical staining. To better understand possible mechanisms of altered pancreas size, we measured pancreas size in three mouse models of insulin deficiency.

Results Pancreases from donors with type 1 diabetes were approximately $45 \%$ smaller than those from donors without diabetes $(47.4 \pm 2.6$ vs $85.7 \pm 3.7 \mathrm{~g})$, independent of diabetes duration or age of onset. Diabetic donor pancreases had decreased beta cell mass $(0.061 \pm 0.025 \mathrm{vs} 0.94 \pm 0.21 \mathrm{~g})$ and reduced total exocrine mass $(42.0 \pm 4.9 \mathrm{vs} 96.1 \pm 6.5 \mathrm{~g})$. Diabetic acinar cells were similar in size but fewer in number compared with those in pancreases from non-diabetic donors $\left(63.7 \pm 8.1 \times 10^{9} \mathrm{vs} 121.6 \pm 12.2 \times 10^{9}\right.$ cells/pancreas), likely accounting for the difference in pancreas size. Within the type 1 diabetes exocrine tissue, there was a greater degree of fibrosis. The pancreases in three mouse models of insulin deficiency were similar in size to those in control mice.

Conclusions/interpretation Pancreases from donors with type 1 diabetes are smaller than normal donor pancreases because exocrine cells are fewer in number rather than smaller in size; these changes occur early in the disease process. Our mouse data suggest that decreased pancreas size in type 1 diabetes is not directly caused by insulin deficiency, but the precise mechanism responsible remains unclear.
\end{abstract}

Keywords Acinar cells $\cdot$ Atrophy $\cdot$ Exocrine $\cdot$ Fibrosis $\cdot$ Histology $\cdot$ Pancreas $\cdot$ Pathogenesis $\cdot$ Type 1 diabetes

\section{Introduction}

The normal adult pancreas is composed of exocrine tissue, constituting approximately $98 \%$ of pancreatic mass, with interspersed endocrine islets composed of beta, alpha and

Electronic supplementary material The online version of this article (https://doi.org/10.1007/s00125-020-05155-y) contains peer-reviewed but unedited supplementary material, which is available to authorised users.

Marcela Brissova

marcela.brissova@vumc.org

Alvin C. Powers

al.powers@vumc.org

1 Division of Diabetes, Endocrinology, and Metabolism, Department of Medicine, Vanderbilt University Medical Center, 7465 Medical Research Bldg IV, 2215 Garland Avenue,

Nashville, TN 37232-0475, USA

2 The Jackson Laboratory, Bar Harbor, ME, USA delta cells responsible for producing insulin, glucagon and somatostatin, respectively.

Type 1 diabetes is caused by immune-mediated destruction of pancreatic beta cells. Recent imaging studies have shown that individuals with newly diagnosed diabetes have a marked
3 Division of Diabetes, Department of Medicine, Diabetes Center of Excellence, University of Massachusetts Medical School, Worcester, MA, USA

4 Institute of Cellular Therapeutics, Allegheny-Singer Research Institute Department of Biological Sciences, Carnegie Mellon University, Pittsburgh, PA, USA

5 Deparment of Molecular Physiology and Biophysics, Vanderbilt University, Nashville, TN, USA

6 Veterans Affairs Tennessee Valley Healthcare System, Nashville, TN, USA 


\section{Research in context}

\section{What is already known about this subject?}

- Pancreases from individuals with type 1 diabetes are smaller than those from non-diabetic donors

\section{What is the key question?}

- What is the cell composition and structure of the diabetic exocrine pancreas?

\section{What are the new findings?}

- Pancreases are smaller in individuals with type 1 diabetes because there are fewer acinar cells. Acinar cell size is unchanged

- Pancreases in individuals with type 1 diabetes have more pancreatic fibrosis

\section{How might this impact on clinical practice in the foreseeable future?}

- Type 1 diabetes is a disease of the entire pancreas, with changes in endocrine and exocrine compartments. This has implications for our understanding of the pathogenesis of type 1 diabetes; how these changes affect pancreatic function should be examined

reduction in pancreas volume $[1,2]$ that is present prior to diagnosis and progressively declines during the first year after diagnosis. Whether this represents changes in exocrine cell size or number or in the extracellular matrix has not been evaluated.

To define the changes that contribute to smaller pancreas, we examined pancreatic endocrine and exocrine tissue from adult donors with type 1 diabetes, and we evaluated pancreas size in three mouse models of insulin deficiency.

\section{Methods}

For detailed methods, please refer to the electronic supplementary material (ESM).

Donor information Human pancreases (31 from donors with type 1 diabetes and 36 from donors without diabetes) and redacted donor characteristics were obtained through partnerships with multiple donor organisations, as previously described [3, 4] (ESM Tables 1 and 2 and ESM Human Islet Checklist).

Immunohistochemical analysis Immunofluorescent staining for endocrine hormones, exocrine markers, extracellular matrix, and markers for apoptosis or cell proliferation was performed on $5 \mu \mathrm{m}$ cryosections of donor pancreases, as previously described [3, 4]. Tissue fibrosis was assessed by Masson's trichrome blue staining on $5 \mu \mathrm{m}$ formalin-fixed paraffinembedded sections (ESM Tables 3 and 4, ESM Fig. 1).

Mouse pancreas size Pancreas and body weight were measured in diabetic and non-diabetic NOD female mice at
17-18 weeks [5], in male Akita (C57BL/6-Ins $\left.2^{\text {Akita }} / \mathrm{J}\right)$ mice at 5 and 12 weeks of age [6], and in NOD.CgPrkd ${ }^{\text {scid } I l 2} \operatorname{rg}^{t m / W^{W j}} \mathrm{Sz}$ (NSG) mice expressing diphtheria toxin receptor under the control of the rat insulin promoter (NSG-DTR, 14-16 weeks). These NSG-DTR mice were part of previously reported studies [7].

Study approval Vanderbilt University Institutional Review Board does not classify studies of de-identified human pancreatic specimens as human subject research and has approved all studies reported herein. All animal studies were approved by the Institutional Animal Care and Use Committee (IACUC) at Vanderbilt University Medical Center or The Jackson Laboratory. Mice were housed and cared for according to the Vanderbilt or Jackson Laboratory Department of Animal Care and IACUC/Office of Animal Welfare Assurance standards and guidelines.

Statistical analysis Data are expressed as mean \pm SEM unless otherwise indicated. Significance was determined using unpaired Student's $t$ test corrected for multiple comparisons using the Holm-Šidák method when appropriate, except for islet size comparisons, which used the Mann-Whitney $U$ test for non-Gaussian distribution of data.

\section{Results}

We collected and studied the pancreas from donors with type 1 diabetes and similarly aged donors without diabetes. Pancreases from donors with diabetes were $45 \%$ smaller than those from donors without diabetes (Fig. 1a, $47.4 \pm 2.6$ vs $85.7 \pm 3.7 \mathrm{~g}, n=31$ and $n=36$, respectively, $p<0.001)$. This 

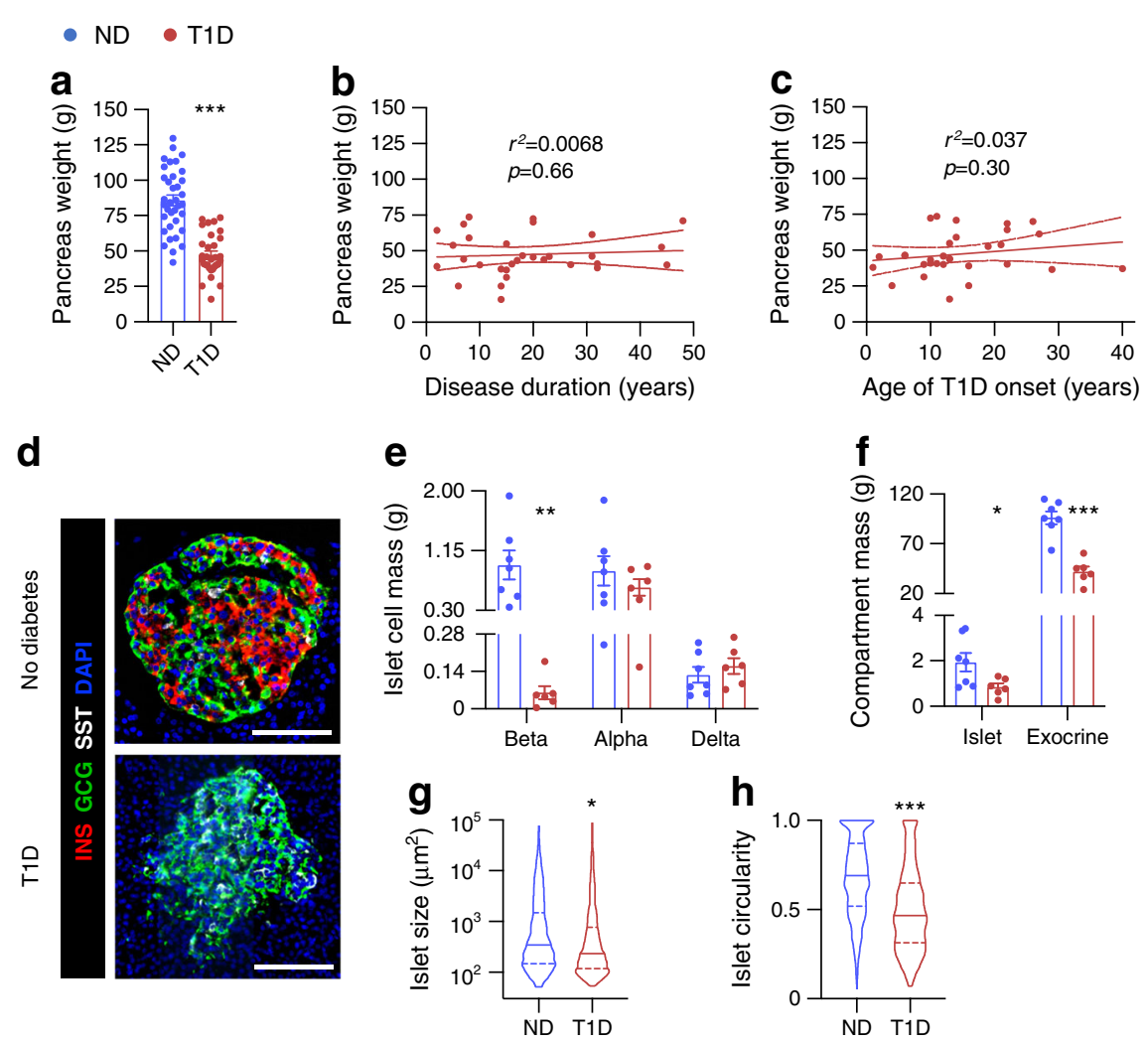

Fig. 1 Pancreas size, endocrine and exocrine mass, and islet morphology differ in type 1 diabetes. (a) Mean pancreas weight of all adult donors ( $>18$ years old); $n=31$ donors with type 1 diabetes (T1D), $n=36$ nondiabetic donors (ND). (b, c) Pancreas weight of adult T1D donors vs duration of diabetes or age of type 1 diabetes onset. INS, insulin; GCG, glucagon; SST, somatostatin. (d) Representative immunofluorescent staining of pancreas from ND and T1D human pancreas. (e) Quantification of beta, alpha and delta cell mass and (f) islet and exocrine compartment mass from $n=6 \mathrm{~T} 1 \mathrm{D}$ and $n=7 \mathrm{ND}$ donors. Islet size (g) and circularity $(\mathbf{h})$, a measure of roundness of islets (where a perfect circle

has a circularity of 1), calculated and pooled from $n=1321$ and $n=719$ islets from $11 \mathrm{ND}$ and 10 T1D donors, respectively. For islet morphology measurements, only islets $>1000 \mu \mathrm{m}^{2}$ were used. Bar graphs represent mean \pm SEM for all panels. For (b) and (c), solid lines represent the line of best fit \pm SEM, and for (g) and (h), lines represent quartiles. The $p$ values were calculated by unpaired Student's $t$ test in $(\mathbf{a}, \mathbf{e}, \mathbf{f})$ and by MannWhitney $U$ test in $(\mathbf{g}, \mathbf{h})$ for non-Gaussian distribution. In (b) and (c) the $p$ value represents deviation of slope of line of best fit from zero. ${ }^{*} p<0.05$, ${ }^{* *} p<0.01, * * * p<0.001 \mathrm{vs}$ ND. Scale bars, $100 \mu \mathrm{m}$

difference was noted whether comparing absolute pancreas weight or relative pancreas weight (normalised to body weight) and did not correlate with donor age, disease duration or age of diabetes onset (Fig. 1b,c, ESM Fig. 2).

To better understand the structural changes in the diabetic donor pancreas, we quantified changes in both the endocrine and exocrine compartments from diabetic $(n=6)$ and nondiabetic $(n=7)$ donors. Total islet mass was $55 \%$ lower in diabetic donor pancreases than in pancreases from nondiabetic donors (Fig. 1f, $0.85 \pm 0.16$ vs $1.9 \pm 0.41 \mathrm{~g}$, respectively, $p=0.04$ ), attributable to decreased beta cell mass (Fig. $1 \mathrm{e}, 0.061 \pm 0.025$ vs $0.94 \pm 0.21 \mathrm{~g}$, respectively, $p=0.007$ ). Absolute alpha and delta cell masses were unchanged (Fig. $1 \mathrm{e})$; alpha and delta cell per cent cross-sectional areas were increased, but the increase was only significant $(p<0.05)$ for delta cells (ESM Fig. 3). Islets from donors with type 1 diabetes were slightly smaller (Fig. 1g) and were irregularly shaped compared with islets from donors without diabetes (Fig. 1h). Total exocrine mass was reduced by $45 \%$ (Fig. 1f, $42.0 \pm 4.9$ vs $96.1 \pm 6.5 \mathrm{~g}, p<0.0001)$. Thus, the pancreas in type 1

diabetes is characterised by reductions in beta cell mass, islet mass, islet size and exocrine mass, while alpha and delta cell mass are unchanged.

Since decreased cell size or number could lead to reduced pancreas size, we evaluated acinar cell size and total cell number. Acinar cells were similar in size between diabetic $(n=10)$ and non-diabetic $(n=11)$ donors regardless of pancreas region (head, body or tail) or intralobular location (centre, periphery or peri-islet) (Fig. 2c,d). Average acinar cell size did not correlate with pancreas weight (Fig. 2e). In contrast, pancreases from donors with type 1 diabetes had $57 \%$ fewer estimated total exocrine cells (Fig. 2f, 63.7 \pm $8.1 \times 10^{9}$ vs $121.6 \pm 12.2 \times 10^{9}$ cells, $p=0.001$ ). To determine if the reduced cell number was due to ongoing changes in cell death or replication, we assessed TUNEL- and Ki67-positive cells in exocrine tissue. Replicating or apoptotic acinar cells were both rare, and the proportions of these cells were similar in diabetic and non-diabetic donors (ESM Fig. 4). The sample size is small, and we cannot definitively exclude increased apoptosis, but TUNEL positivity did not correlate with a 


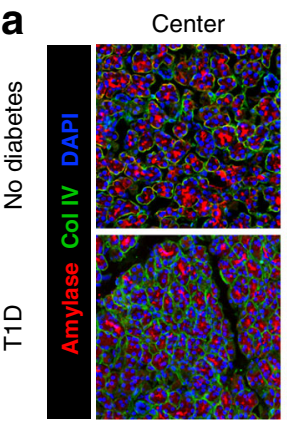

C

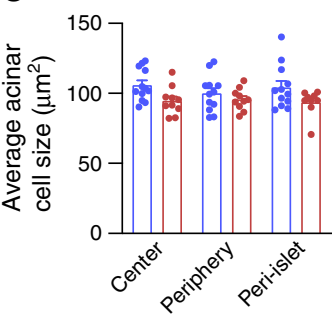

d
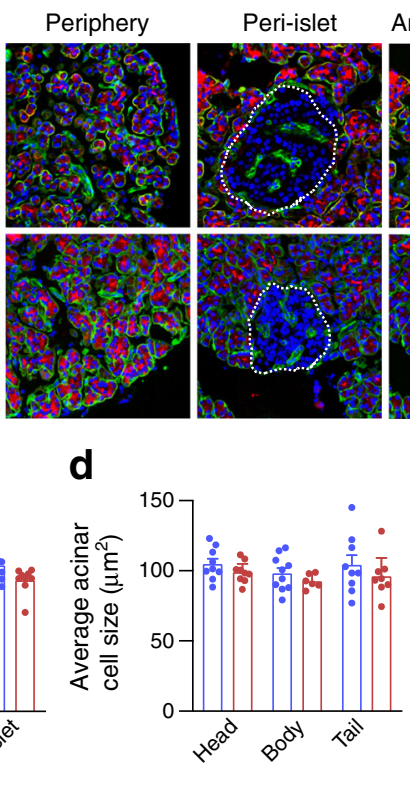

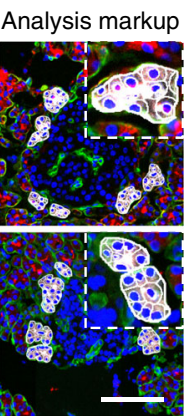

e

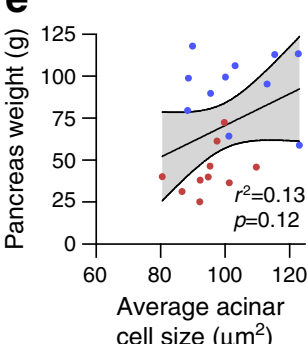

- ND

- T1D

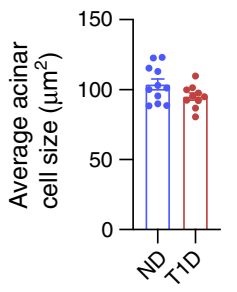

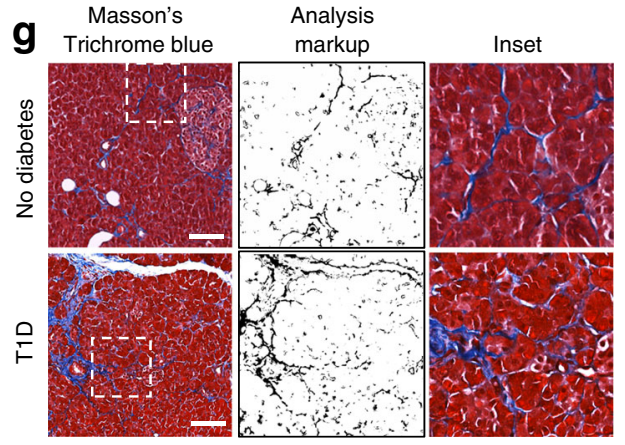

Fig. 2 Pancreas from donors with type 1 diabetes has altered acinar cell number and extracellular matrix. (a) Representative images of sections from indicated pancreatic lobe regions. Individual acini were manually outlined, cells counted based on DAPI staining, and average acinar cell size calculated by dividing acinar area by cell number. Islet area is denoted by the dotted line. Examples of outlined acini with counted cells are shown in analysis markup panels. Col IV, collagen IV. Acinar cell size is shown averaged across entire pancreas (b), separated by location within pancreatic lobes (c) and by region of pancreas (d). (e) Acinar cell size vs pancreas weight. (f) Estimated total cells per pancreas was calculated by

decrease in pancreas size. Taken together, these data indicate that pancreases in individuals with type 1 diabetes have fewer acinar cells, but acinar cell size, proliferation and apoptosis are similar to those in pancreases in non-diabetic individuals.

In processing the diabetic donor pancreas, we also noted that the organs had a firmer texture. Further to this observation and because changes in exocrine extracellular space could contribute to changes in pancreas size, we quantified fibrosis in the pancreases using trichrome blue staining. The diabetic donor pancreases had more fibrosis, which correlated negatively with pancreas size (Fig. 2g-i). Qualitatively, increased fibrosis in diabetic donor pancreases was observed in two patterns: thickening of the small inter-acinar septa, and dense intraparenchymal h
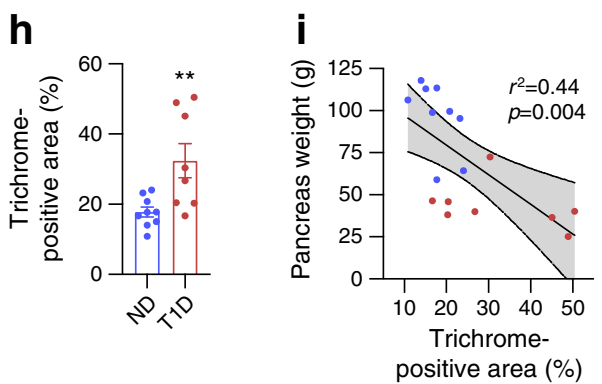

multiplying the cell density (cells per $\mathrm{mm}^{3}$ ) by pancreas weight. For $(\mathbf{b}-\mathbf{f})$ $n=10$ type 1 diabetic donors (T1D) and 11 non-diabetic donors (ND). (g) Pancreas fibrosis was measured after Masson's trichrome stain in $n=8$ T1D and $n=9$ ND pancreases, showing analysis markup and highmagnification inset. Trichrome positive area (h) correlated with pancreas weight (i). Bar graphs are mean \pm SEM for all panels. For (e) and (i), solid lines represent best fit line $\pm 95 \%$ CI. The $p$ values are calculated by unpaired Student's $t$ test, except for (e) and (i), where the $p$ value represents deviation of line of best fit from zero. ${ }^{* *} p<0.01$ vs ND. Scale bars, $100 \mu \mathrm{m}$

deposition of connective tissue (often near islets). Thus, exocrine changes in longstanding diabetes involve both cellular and extracellular compartments.

Finally, to investigate whether reduced insulin production in diabetes might be responsible for the reduced pancreatic mass, we quantified pancreas weight in three mouse models with markedly reduced insulin production: NOD, Akita and NSG-DTR, which are models of autoimmune islet destruction, misfolded mutant proinsulin-induced toxicity and toxin-mediated beta cell death, respectively. In all three models, pancreas weight was unchanged in diabetic mice compared with control mice (ESM Fig. 5). Thus, in mice, loss of insulin or beta cells does not lead to reduced pancreatic weight. 


\section{Discussion}

In individuals with type 1 diabetes the pancreas is approximately half the size of that in non-diabetic individuals, but the nature of this change is poorly understood. By studying human pancreatic tissue, we found qualitative and quantitative changes in the endocrine and exocrine pancreas. In the exocrine pancreas, there was a marked decrease in acinar cell number and increased fibrosis, with no change in acinar cell size, proliferation or apoptosis. The endocrine compartment had reduced beta cell mass with slightly smaller and dysmorphic islets but unchanged alpha or delta cell mass.

Pancreas volume progressively declines during the first year of type 1 diabetes [1], and first-degree relatives of individuals with diabetes have pancreas volumes intermediate between those of diabetic and control individuals [2]. In the current study, we found that pancreas size did not vary with duration of disease, supporting the concept that loss of pancreatic mass occurs both prior to, and relatively early after, clinical disease onset. Longitudinal imaging studies of individuals over a range of diabetes durations and of their high-risk family members will clarify the timing of these changes in pancreas size. It is possible that this metric could be useful in defining risk in diabetes intervention and prevention clinical trials.

The mechanism responsible for reduced acinar cell number in diabetes is not clear. It is possible that acinar cells are affected by the autoimmune attack in type 1 diabetes, possibly through cytokine-, cell- or antibody-mediated mechanisms $[8,9]$. On the other hand, early studies of cadaveric pancreas from donors with recently diagnosed diabetes found exocrine atrophy surrounding inflamed or insulin-deficient islets [10], suggesting that local paracrine signalling may contribute to changes in exocrine tissue. We did not see these changes in diabetes of longer duration. Other possibilities, including alterations in blood flow to exocrine tissue or altered developmental processes leading to decreased pancreas size prior to disease onset, have not been studied extensively in human tissue.

Another potential mechanism is that loss of beta cellderived trophic factors leads to exocrine cell loss. Indeed, some peri-islet acini exhibit alterations in amylase expression, suggesting an islet-acinar axis [11]. Our results suggest that changes in pancreas size are not caused by loss of beta cellderived paracrine growth factors. First, we observed no differences in acinar cell size, proliferation or apoptosis regardless of proximity to islets. Second, pancreas size did not differ between donors with childhood- or adult-onset diabetes unlike what would be expected if a critical exocrine growth factor were missing during the first two decades of life when the exocrine pancreas grows most rapidly. Third, acknowledging inter-species differences in islet structure, function and pathology, the mouse data from three different models with similarities to human type 1 diabetes did not show that insulin deficiency leads to a reduction in pancreas size. Further studies of early-onset diabetic tissue are required to better delineate the mechanisms of exocrine cell loss.

These studies indicate that type 1 diabetes affects both the exocrine and the endocrine compartments of the pancreas, which is supported by clinical studies showing mild exocrine deficiency in type 1 diabetes [12]. Elucidation of the timing and mechanism of changes in acinar cell number may contribute to improved understanding of disease pathogenesis.

Acknowledgements We thank the organ donors and their families for their invaluable donation and the International Institute for the Advancement of Medicine (IIAM), organ procurement organisations, the Allegheny-Singer Research Institute, the National Disease Research Interchange (NDRI), the Network for Pancreatic Organ Donors with Diabetes (nPOD), and the Alberta Diabetes Institute Islet Core for their partnership in studies of human pancreatic tissue for research. We thank the Vanderbilt University Translational Pathology Shared Resource for assistance with trichrome blue staining.

Data availability The datasets generated and/or analysed during the current study are available from the corresponding author. Images of stained tissue sections used in this manuscript are available at www. pancreatlas.org/datasets/703/overview.

Funding This research was performed using resources and/or funding provided by the National Institute of Diabetes and Digestive and Kidney Diseases-supported Human Islet Research Network and the Human Pancreas Analysis Program (RRID: SCR 014393; https:// hirnetwork.org; UC4 DK104211, DK108120, and DK112232), by DK106755, DK72473, DK89572, DK97829, DK94199, T32DK7061, DK46266, DK95735, OD020351 and DK20593, The Leona M. and Harry B. Helmsley Charitable Trust, and the Department of Veterans Affairs (BX000666). This research used resources from the Network for Pancreatic Organ donors with Diabetes (nPOD; RRID:SCR 014641), a collaborative type 1 diabetes research project sponsored by JDRF (nPOD: 5-SRA-2018-557-Q-R). The content and views expressed are the responsibility of the authors and do not necessarily reflect the official view of nPOD. Organ Procurement Organizations (OPO) partnering with nPOD to provide research resources are listed at http:// www.jdrfnpod.org/for-partners/npod-partners/.

Contribution statement JJW, DCS, DMH, RB, MB and ACP contributed substantially to the conception and design of the reported experiments. JJW, CD, GP, BC, RB and DVS contributed to the acquisition of data, and JJW, DCS, CD, DVS, MB and ACP contributed to the analysis and interpretation of the data. JJW and ACP drafted the initial article, and all co-authors contributed to revising it for intellectual content. All co-authors have given final approval of the published version. As guarantor of this work, ACP accepts full responsibility for the work and/or the conduct of the study, had access to the data, and controlled the decision to publish.

\section{References}

1. Virostko J, Williams J, Hilmes M et al (2019) Pancreas volume declines during the first year after diagnosis of type 1 diabetes and exhibits altered diffusion at disease onset. Diabetes Care 42(2):248-257. https://doi.org/10.2337/dc18-1507

2. Campbell-Thompson ML, Filipp SL, Grajo JR et al (2019) Relative pancreas volume is reduced in first-degree relatives of patients with 
type 1 diabetes. Diabetes Care 42(2):281-287. https://doi.org/10. 2337/dc18-1512

3. Haliyur R, Tong X, Sanyoura M et al (2019) Human islets expressing HNF1A variant have defective beta cell transcriptional regulatory networks. J Clin Invest 129(1):246-251. https://doi.org/10. 1172/JCI121994

4. Brissova M, Haliyur R, Saunders D et al (2018) $\alpha$ cell function and gene expression are compromised in type 1 diabetes. Cell Rep 22(10):2667-2676. https://doi.org/10.1016/j.celrep.2018.02.032

5. Presa M, Racine JJ, Dwyer JR et al (2018) A hypermorphic Nfkbid allele contributes to impaired thymic deletion of autoreactive diabetogenic CD8 ${ }^{+}$T cells in NOD mice. J Immunol 201(7):1907-1917. https://doi.org/10.4049/jimmunol.1800465

6. Wang J, Takeuchi T, Tanaka S et al (1999) A mutation in the insulin 2 gene induces diabetes with severe pancreatic beta-cell dysfunction in the Mody mouse. J Clin Invest 103(1):27-37. https://doi.org/ 10.1172/JCI4431

7. Dai C, Kayton NS, Shostak A et al (2016) Stress-impaired transcription factor expression and insulin secretion in transplanted human islets. J Clin Invest 126(5):1857-1870. https://doi.org/10. 1172/JCI83657

8. Rodriguez-Calvo T, Ekwall O, Amirian N, Zapardiel-Gonzalo J, von Herrath MG (2014) Increased immune cell infiltration of the exocrine pancreas: a possible contribution to the pathogenesis of type 1 diabetes. Diabetes 63(11):3880-3890. https://doi.org/10. 2337/db14-0549

9. Panicot L, Mas E, Thivolet C, Lombardo D (1999) Circulating antibodies against an exocrine pancreatic enzyme in type 1 diabetes. Diabetes 48(12):2316-2323. https://doi.org/10.2337/diabetes. 48.12.2316

10. Foulis AK, Stewart JA (1984) The pancreas in recent-onset type 1 (insulin-dependent) diabetes mellitus: insulin content of islets, insulitis and associated changes in the exocrine acinar tissue. Diabetologia 26(6):456-461. https://doi.org/10.1007/bf00262221

11. Kusmartseva I, Beery M, Hiller H et al (2020) Temporal analysis of amylase expression in control, autoantibody positive, and type 1 diabetes pancreatic tissues. Diabetes 69(1):60-66. https://doi.org/ $10.2337 / \mathrm{db} 19-0554$

12. Campbell-Thompson M, Rodriguez-Calvo T, Battaglia M (2015) Abnormalities of the exocrine pancreas in type 1 diabetes. Curr Diab Rep 15(10):79. https://doi.org/10.1007/s11892-015-0653-y

Publisher's note Springer Nature remains neutral with regard to jurisdictional claims in published maps and institutional affiliations. 